\title{
Neuroleptic malignant syndrome cases in a Moroccan intensive care unit: a retrospective analysis and literature review
}

\author{
Soumaya Touzani, MD; Mohammed Hamdouni, MD; Nawfal Houari, MD; \\ Youssef Yaakoubi Khbiza, MD; Abderrahim Elbouazzaoui, MD; \\ Brahim Boukatta, MD; Nabil Kanjaa, MD
}

Anesthesiology and Intensive Care Department A4, Hassan II University Hospital, Sidi Mohammed Ben Abdellah University, Fez, Morocco

\section{Journal of Neurocritical} Care

ORIGINAL ARTICLE

Received: August 20, 2021

Revised: September 30, 2021

Accepted: October 7, 2021

Corresponding Author:

Soumaya Touzani, MD

Anesthesiology and Intensive Care Department A4, Hassan II University Hospital, Sidi Mohammed Ben Abdellah University, Faculty of Medicine and Pharmacy, Sidi Hrazem Rd, Fez 30070, Morocco

E-mail: touzani.soumaya@gmail.com

Background: Neuroleptic malignant syndrome (NMS) is a rare but potentially life-threatening neuropsychiatric emergency. The aim of our study was to update our bedside procedures by investigating NMS cases managed in the intensive care unit (ICU).

Methods: This retrospective study included all NMS patients admitted to our hospital between January 2012 and December 2019. The variables analyzed included demographics, diagnosis, therapeutics, and outcomes.

Results: This study included 20 patients, with an average age of 36.6 years. The male to female ratio was 1:4. No patient had a history of NMS, and $60 \%$ of the patients had schizophrenia. First-generation neuroleptics (NLs) were the most commonly prescribed drugs $(80 \%)$. The mean time between the introduction of NLs and onset of symptoms was 7.6 days. Rigidity was observed in $90 \%$ of the patients, hyperthermia and neuropsychic syndrome in 65\%, and dysautonomia in 50\%. The creatine phosphokinase level in all patients was four times the normal value. Mechanical ventilation was required in $20 \%$ of the patients and hemodialysis in one patient. None of the patients received specific therapy. The mean duration of ICU stay was 10 days. The mortality rate was $10 \%$ " mainly associated with renal failure. The analysis of the predictors of mortality was limited by the size of our cohort.

Conclusion: NMS is a rare condition requiring multidisciplinary implementation of contextualized and updated procedures. Early detection and supportive treatment could improve the prognosis in resource-limited settings, where specific treatments are not available. Predictive risk factors should be investigated in larger multicenter cohorts.

Keywords: Neuroleptic malignant syndrome; Intensive care unit; Antipsychotics; Mortality; Treatment

\section{INTRODUCTION}

Neuroleptic malignant syndrome (NMS) is an idiosyncratic reaction mainly related to the use of antipsychotic agents (first-or second-generation neuroleptics [NLs]). It is characterized by a myriad of clinical signs, including altered mental status, muscular rigid- ity, hyperthermia, and dysautonomia. This life-threatening neuropsychiatric emergency is rare, with an estimated incidence of $0.2 \%$ among NL users [1], and requires early therapeutic management and intensive care for potentially severe presentations. Mortality, caused by dysautonomic and systemic complications (infections, venous thromboembolism, rhabdomyolysis, acute renal failure, 
respiratory failure, etc.), decreased by $76 \%$ since the first reports in the 1960s; the current mortality rate is estimated at $10 \%-20 \%$ $[2,3]$. This is indicative of greater awareness, earlier diagnosis in both emergency settings and psychiatric wards, and interventions that are more aggressive than before. Since NMS requires a high degree of clinical suspicion for diagnosis and treatment, itis rightly a syndrome more often considered in differential diagnosis than is actually diagnosed. Moreover, considering its low incidence, there is limited evidence in the literature. Although the management of NMS is mostly performed in critical care settings, the issue is either not addressed or poorly addressed in the guidelines of the international and national scientific societies of intensive care and emergency medicine. This study aimed to analyze the NMS cases in our hospital to improve clinical decision-making based on the updated and contextualized bedside procedures.

\section{METHODS}

\section{Study design and setting}

This retrospective, observational, monocentric study evaluated patients aged $\geq 16$ years who were admitted to our intensive care unit (ICU) between January 2012 and December 2019. The diagnosis of NMS was based on the diagnostic criteria in the Diagnostic and Statistical Manual of Mental Disorders, 5th edition (DSM-5) of the American Psychiatric Association [4] (Table 1). The diagnosis of NMS was made based on the presence of exposure to a dopaminergic antagonist within the last 72 hours, a suggestive symptomatology, and negative examination results for infectious, toxic, metabolic, and neurologic causes. The study setting was a 14-bed medico surgical adult ICU in a tertiary university hospital in Morocco.

\section{Data collection}

Study data were collected retrospectively from both paper charts and electronic medical records of patients using HOSIX (SIVSA Soluciones Informáticas, Vigo, Spain) electronic data capture tools present at our university hospital. The variables analyzed included demographic characteristics, patient's history and comorbidities, all drugs involved, diagnostic parameters, therapeutics, and evolution.

\section{Statistical analysis}

Statistical analysis of the parameters was performed using IBM SPSS ver. 20 (IBM Corp., Armonk, NY, USA). Descriptive statistics were used to summarize the baseline patient characteristics. The results are expressed as numbers and percentages for qualitative variables and as means \pm standard deviations for the quantitative variables. The quantitative and qualitative variables were compared using the two-sample $t$-test derived from Student $t$ distribution and Fisher's exact test based on the N-1 chi-square test through univariate analysis. The statistical significance threshold was set at a $P$-value of 0.05 . Analysis of predictive factors of mortality through multivariate analysis was not performed because of the limited number of patients in the "death" group, i.e., the group with deceased patients.

Table 1. Diagnostic criteria for neuroleptic malignant syndrome adapted from the Diagnostic and Statistical Manual of Mental Disorders, 5 th edition [4]

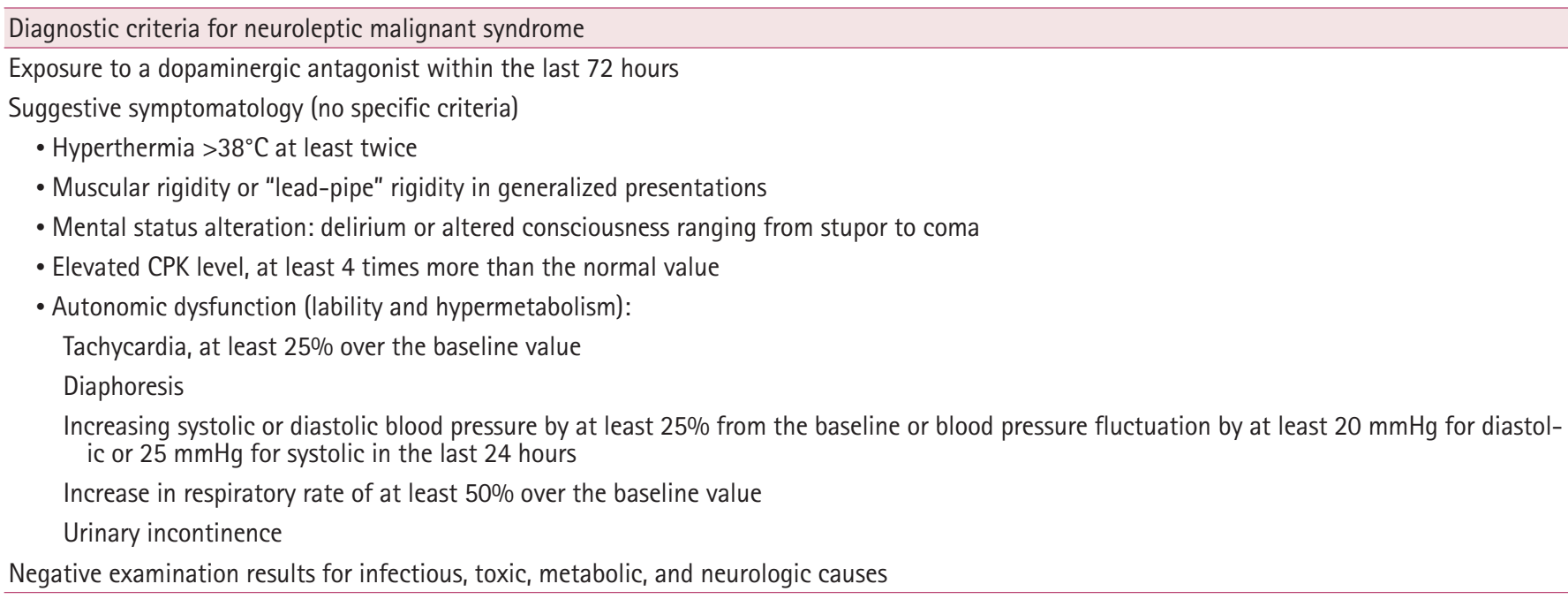

CPK, creatine phosphokinase. 


\section{RESULTS}

This study included 20 patients ( 16 males and 4 females) from the 6,090 patients admitted to our ICU between 2012 and 2019, with a mean age of $36.6 \pm 19.5$ years (range, $18-84$ years). The underlying comorbidities in our population were schizophrenia (60\%), bipolar disorder (20\%), substance dependence (25\%), mental retardation (10\%), acute psychosis (10\%), dementia (5\%), and delirium (5\%). None of the patients had a history of NMS. There were no other medical comorbidities, except for toxic addictions (smoking and cannabism) in five patients and psychomotor disability in two patients. In all the patients, NMS was caused due to the use of antipsychotic drugs. First-generation conventional NLs were the most commonly used drugs ( $80 \%$ of the patients) and mainly administered along with atypical NLs or other drugs (70\% of the patients). Atypical NLs were used in 11 patients (55\%). The medications used in our population included first-generation conventional NLs (haloperidol, 40\%; levomepromazine, $35 \%$; and chlorpromazine, $35 \%$ ), second-generation atypical NLs (risperidone, 25\%; olanzapine, 15\%; and amisulpride, 10\%), and other drugs (selective serotonin reuptake inhibitors [SSRIs], benzodiazepines, and anticholinergics). No case of dopaminergic agonist withdrawal was reported. The parenteral mode of administration was used in only four cases, and $10 \%$ of the patients required a rapid increase in NL doses. The mean time between the introduction of NLs and the onset of symptoms was $7.6 \pm 7.1$ days (range, $0-30$ days). The mean time between symptom onset and hospital admission was $1.3 \pm 1.5$ days (range, $0-5$ days). Rigidity was observed in $90 \%$ of patients, hyperthermia and neuropsychic syndrome in 65\%, and dysautonomia in 50\%. All patients presented a creatine phosphokinase (CPK) level four times more than the normal value. The average CPK was $4,810 \pm 9,789 \mathrm{IU} / \mathrm{L}$, with extremes ranging from 626 to 42,670 IU/L. The average Sachdev rating scale score on admission was $11 \pm 3.09$ (range, 3-14). Examinations for infectious, toxic, metabolic, and neurologic causes were negative in all patients. The distribution of patients according to different diagnostic criteria is presented in Table 2. Psychotropic treatment was stopped in all patients as soon as NMS diagnosis was suspected. Mechanical ventilation was required in $20 \%$ of the patients for a mean duration of 14.75 days (range, 1-28 days). Tracheotomy was performed in two patients on days 4 and 13 of intubation, respectively. All patients underwent respiratory physiotherapy and fluid resuscitation. A continuous intravenous infusion of nicardipine at a dose of 2-6 mg/hr was required in one patient. One patient needed catecholamines, while another needed hemodialysis. Diazepam, midazolam, or clonazepam against agitation were required in $25 \%$ of the patients. Contention was necessary in one patient. No patient was administered dantrolene or bromocriptine, and none received electroconvulsive therapy (ECT). The mean duration of ICU stay was $10 \pm 15$ days (range, 1-67 days). The overall outcome was favorable in $90 \%$ of the patients. The improvements in temperature, rigidity, CPK levels, and renal function varied (Table 3). One case progressed to persistent acute renal failure, classified as Risk; Injury; Failure; Loss; End stage kidney disease (RIFLE) 3. One case of cerebral hemorrhage due to ruptured arteriovenous malformation was recorded. The reintroduction of atypical NLs administered at low doses was conducted after a 15-

Table 2. Distribution of the patients according to different consensus diagnostic criteria

\begin{tabular}{|c|c|c|}
\hline Consensus & Required criteria & Number of patients meeting the criteria (\%) \\
\hline DSM-5 $5^{\text {a) }}$ & No specific criteria & $20(100)$ \\
\hline DSM-IV & Two of criteria $A$ and at least two criteria $B$ and criteria $C$ and $D$ & $11(55)$ \\
\hline Nierenberg et al's diagnostic criteria ${ }^{c}$ & Essential criteria and 4 major or 3 major +3 minor & $13(65)$ \\
\hline Sachdev rating scale ${ }^{d)}$ & Total score $>8$ and a score $\geq 2$ in at least 3 domains & $17(85)$ \\
\hline
\end{tabular}

DSM, Diagnostic and Statistical Manual of Mental Disorders; CPK, creatine phosphokinase.

${ }^{a}$ Hyperthermia, rigidity, mental status alteration, CPK elevation, sympathetic nervous system lability, and hypermetabolism after exposure to dopamine antagonist or dopamine agonist withdrawal, with a negative examination results for infectious, toxic, metabolic, and neurologic causes; ${ }^{\text {bl}} \mathrm{Criteria}_{\mathrm{A}} \mathrm{A}$ muscle rigidity and hyperthermia associated with antipsychotic drugs use; criteria B: diaphoresis, elevated or labile blood pressure, tachycardia, incontinence, dysphagia, mutism, tremor, labile consciousness level ranging from confusion to coma, leukocytosis, and elevated CPK level; criteria C: symptoms in criteria A and B not due to another substance or due to neurologic or other medical conditions; criteria D: symptoms in criteria A and B not accounted for better by a mental disorder; ${ }^{\circ}$ Essential criteria: recent exposure to dopamine antagonist or dopamine agonist withdrawal; major criteria: fever $>38^{\circ} \mathrm{C}$ without other causes, muscular lead-pipe rigidity, elevated serum CPK level ( $>3$ times the normal value without any other cause), autonomic instability (two or more symptoms of sweating, tachycardia, and elevated or decreased blood pressure), and altered consciousness; minor criteria: other autonomic dysfunction (urinary incontinence, arrhythmias, or any one of sweating, tachycardia, and elevated or decreased blood pressure), other extrapyramidal signs (tremor, cog-wheeling, acute dystonic reaction, or choreiform movements), respiratory problems (severe dyspnea, tachypnea, respiratory failure, or hypoxemia), and leukocytosis; ${ }^{\mathrm{d})}$ I: oral temperature; II: extrapyramidal symptoms (rigidity, dysphagia, and resting tremor); III: autonomic instability (increased systolic blood pressure, increased diastolic blood pressure, tachycardia, diaphoresis, incontinence, and tachypnea); IV: altered consciousness; V: catatonia/ movement disorder; and VI: biology (elevated CPK level and leukocytosis). 
Table 3. Clinical and biological evolution of neuroleptic malignant syndrome cases

\begin{tabular}{lcc}
\hline Improved parameter & Number of patients $(n=20)$ & Mean time to improvement since NL discontinuation \\
\hline Temperature $\left(\leq 38^{\circ} \mathrm{C}\right)$ & $20(100)$ & $3.8 \pm 1.3(1-5)$ \\
No rigidity & $18(90)$ & $5.2 \pm 2.2(2-11)$ \\
CPK level $\leq 4$ times normal value & $14(70)$ & $5.9 \pm 2.3(3-10)$ \\
Improved renal function & $17(85)$ & $4.3 \pm 1.5(3-6)$ \\
\hline
\end{tabular}

Values are presented as number (\%) or mean \pm standard deviation (range). $\mathrm{NL}$, neuroleptic; CPK, creatinine phosphokinase.

day therapeutic interval in six patients (30\%). No adverse effects were observed. The mortality rate was $10 \%$, and the deaths were associated with renal failure complications. Two patients developed metabolic arrhythmias, mainly induced by refractory hyperkalemia and exacerbated by severe rhabdomyolysis. The mean time from ICU admission to death was $4 \pm 1.41$ days (range, $3-5$ days). When comparing the "death" ( $\mathrm{n}=2)$ and "recovery" $(\mathrm{n}=18)$ groups, univariate analysis identified nine variables that were significantly $(P<0.05)$ associated with mortality in NMS in our population (Table 4). The analysis of predictive factors of mortality through multivariate analysis was not possible given the limited number of patients in the "death" group.

\section{DISCUSSION}

This study, comprising 20 cases, is one of the largest cohorts of NMS patients in Morocco and one of the few studies conducted in ICUs. The incidence of NMS among NL users varies between $0.024 \%$ and $3 \%$ [5]. This is difficult to assess accurately due to population heterogeneity, variability in diagnostic criteria, and the methodological limitations of retrospective studies. In our study, NMS was predominant in young adult males, as similarly reported in the literature [6].

This can be explained by the higher skeletal muscle mass in males, and therefore more visible symptoms and more severe forms [7]; higher frequency of schizophrenia in males, and therefore a higher need for antipsychotics at elevated doses [8]; and sexual dimorphism in dopaminergic pathways, as suggested in recent studies [9].

NMS was first described in 1960 with haloperidol and was labeled as malignant by the Parisian group of the Sainte-Anne Hospital, analogous with the malignant syndrome of infectious diseases widely prevalent in the 1950s and 1960s [10].

However, any drug interfering with dopaminergic transmission can lead to NMS [11]. Combination of antipsychotic drugs, combined use of an antipsychotic with lithium or carbamazepine, abrupt discontinuation of a dopaminergic agonist such as levodopa in Parkinson disease patients, and the use of antiemetics such as metoclopramide have been reported to induce NMS. The combined use of several therapeutic classes of drugs seems to be associated with a higher risk of NMS, despite the higher affinity of conventional antipsychotics for dopaminergic D2 receptors $[12,13]$. In our series, no cases of dopaminergic agonist discontinuation were reported, and conventional NLs often combined with atypical NLs were most commonly used. Recently, more researchers have suggested "malignant extrapyramidal autonomic syndrome" diagnosis instead of NMS [14] to improve proactive screening in the absence of the use of antipsychotic drugs. The risk of NMS is higher within the first month of treatment at high doses, especially when administered parenterally or after a rapid dose change. Physical restraint during psychomotor agitation is often associated with high titration rates and parenteral therapies, and therefore increases the risk of NMS [15]. In our population, NMS occurred within a mean interval of 7 days (range, 24 hours-30 days), all doses were standard, and the parenteral route was only used in $20 \%$ of the cases. Therefore, NMS can occur at any time during treatment, even with standard doses, and regardless of the route of administration. Other risk factors have been reported, such as advanced age, comorbid medical conditions, mental retardation, history of NMS, and personal and/or family history of catatonia [16].

Regardless of the trigger mechanism (dopaminergic antagonism, dysautonomia, direct muscle toxicity of NL, etc.), the physiopathology of NMS is complex and involves a cascade of dysfunctions in multiple neurochemical and neuroendocrine systems, leading to end-stage hypermetabolic syndrome [8,17-19]. NMS is classically [1] characterized by four cardinal signs: hyperthermia, muscular rigidity, dysautonomia, and altered mental status. Neuropsychic syndrome usually precedes the systemic symptoms. Muscle rigidity can be generalized and could be symmetric (opisthotonos) or focal (blepharospasm, oculogyric crisis, and trismus). NMS hyperthermia usually presents high body temperatures, with no major peaks or fluctuations, no shivering, and unresponsiveness to conventional antipyretics. However, the clinical presentation of NMS can be heterogeneous and challenging (Table 5) [13,20-23]. Cases of NMS without muscle rigidity have 
Table 4. Univariate analysis of risk factors for mortality in the NL malignant syndrome cases $(n=20)$

\begin{tabular}{|c|c|c|c|}
\hline Parameter & Death $(n=2)$ & Recovery $(n=18)$ & $P$-value \\
\hline Age (yr) & $71.5 \pm 16.3(60-83)$ & $32.7 \pm 15.8(18-84)$ & 0.004 \\
\hline Male sex & $2(100)$ & $14(78)$ & 0.630 \\
\hline Mean time to consultation (day) & $0.5 \pm 0.7(0-1)$ & $1.5 \pm 1.5(0-5)$ & 0.360 \\
\hline Admission from domicile & $1(50)$ & $5(27.7)$ & 0.520 \\
\hline Duration of NL treatment (day) & $7.0 \pm 1.4(6-8)$ & $9.1 \pm 7.9(1-30)$ & 0.710 \\
\hline GCS on admission & 13.00 & $13.64 \pm 1.49(10-15)$ & \\
\hline Heart rate on admission (beats/min) & $85.00 \pm 7.07(80-90)$ & $98.88 \pm 24.92(56-140)$ & 0.450 \\
\hline Diastolic blood pressure $(\mathrm{mmHg})$ & $80.00 \pm 14.14(70-90)$ & $78.05 \pm 11.77(60-100)$ & 0.450 \\
\hline Systolic blood pressure $(\mathrm{mmHg})$ & $130.00 \pm 14.14(120-140)$ & $138.61 \pm 24.24(90-200)$ & 0.630 \\
\hline Temperature $\left({ }^{\circ} \mathrm{C}\right)$ & $37.65 \pm 0.92(37-38.3)$ & $38.11 \pm 1.06(35-39)$ & 0.560 \\
\hline Dysautonomia & 0 & $13(72.2)$ & 0.110 \\
\hline Rigidity & $2(100)$ & $16(88.8)$ & 0.800 \\
\hline Quick SOFA score & $1.00 \pm 1.00(0-2)$ & $0.88 \pm 0.75(0-2)$ & 0.830 \\
\hline Sachdev rating scale & $12.50 \pm 1.50(11-14)$ & $11.00 \pm 3.19(3-14)$ & 0.520 \\
\hline CPK (IU/L) & $\begin{array}{c}21,964.00 \pm 29,282.70 \\
42,670)\end{array}$ & $2,904.00 \pm 4,265.86(626-19,176)$ & 0.005 \\
\hline CPK-mb (IU/L) & $647.50 \pm 846.40(49-1,246)$ & $86.72 \pm 71.45(24-327)$ & 0.002 \\
\hline WBC count $\left(\times 10^{3} / \mathrm{mm}^{3}\right)$ & $9.70 \pm 1.97(8.38-11.17)$ & $11.31 \pm 5.04(4.89-25)$ & 0.660 \\
\hline AST (IU/L) & $366.00 \pm 376.18(100-632)$ & $71.22 \pm 62.84(17-296)$ & 0.002 \\
\hline ALT (IU/L) & $148.50 \pm 136.47(52-245)$ & $41.72 \pm 33.71(10-155)$ & 0.006 \\
\hline Creatinine (mg/L) & $102.00 \pm 134.35(7-197)$ & $8.55 \pm 2.22(5-14)$ & 0.001 \\
\hline Blood urea nitrogen (mg/L) & $2.45 \pm 3.02(0.29-4.60)$ & $0.33 \pm 0.28(0.09-1.23)$ & 0.002 \\
\hline Prothrombin (\%) & $56.50 \pm 9.19(50-63)$ & $87.05 \pm 8.72(69-100)$ & 0.000 \\
\hline Platelet $\left(\times 10^{3} / \mathrm{mm}^{3}\right)$ & $97.00 \pm 29.69(76-118)$ & $246.05 \pm 78.25(147-444)$ & 0.017 \\
\hline Blood potassium (mmol/L) & $4.80 \pm 2.80(2.8-6.8)$ & $3.86 \pm 0.54(2.7-5.1)$ & 0.152 \\
\hline NL discontinuation time (day) & $2.0 \pm 1.4(1-3)$ & $2.9 \pm 1.3(0-6)$ & 0.367 \\
\hline Mechanical ventilation & $2.0(100)$ & $2(11.11)$ & 0.030 \\
\hline Hemodialysis & $1(50)$ & 0 & 0.100 \\
\hline Pulmonary infection & 0 & $3(16.6)$ & 0.710 \\
\hline Thromboembolic complication & 0 & 0 & \\
\hline ICU stay (day) & $3.50 \pm 2.12(2-5)$ & $10.83 \pm 16.11(1-67)$ & 0.538 \\
\hline
\end{tabular}

Values are presented as mean \pm standard deviation (range) or number (\%).

NL, neuroleptic; GCS, Glasgow coma scale; SOFA, Sequential Organ Failure Assessment; CPK, creatine phosphokinase; CPK-mb, creatine phosphokinasemyoglobin binding; WBC, white blood cell; AST, aspartate transaminase; ALT, alanine aminotransferase; ICU, intensive care unit.

been reported in the literature $[24,25]$ as well as in this study ( $10 \%$ of the sample). Other extrapyramidal motor symptoms, such as tremor, chorea, akinesia, mutism, dysarthria, dysphagia, and other dystonic movements, may guide the diagnosis, but they are inconstant and non-specific. Approximately $20 \%$ of the patients had dysphagia or mutism, while hyperthermia was reported in only $65 \%$. Apyretic NMS cases can be explained by the early diagnosis of NMS and/or a delayed onset of hyperthermia when compared to early symptoms such as rigidity [21]. Non-typical presentations are mostly associated with atypical NLs [26]. This is consistent with our study findings, where in the absence of rigidity and hyperthermia were observed respectively in $25 \%$ and $50 \%$ of the cases using atypical NLs compared to $0 \%$ and $33.3 \%$ of the cases using conventional NLs.

Although no biomarkers are specific, laboratory assessment is necessary to support the diagnosis of NMS by excluding other diagnoses and to assess complications. Rigidity and hyperthermia lead to muscle damage and rhabdomyolysis (elevated CPK levels and myoglobinemia) with a risk of hyperkalemia, hyperphosphatemia, hyperuricemia, hypocalcemia, heart rhythm disturbances, disseminated intravascular coagulation, and renal failure [27]. The CPK level is considered both a diagnostic and prognostic marker of NMS (especially if CPK > 1,000 IU/L), as well as a monitoring factor to assess the course and effectiveness of the treatment (kinetics). A mild or no increase in the CPK level may be idiopathic or related to the early stages of NMS, where rigidity 


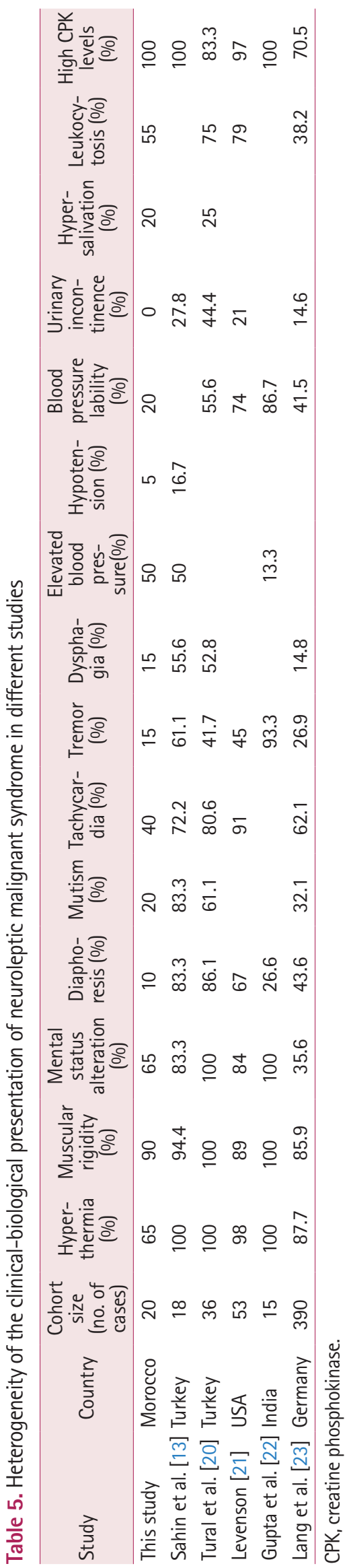

is absent or poorly developed and maybe associated with physical restraint and intramuscular injections, especially in catatonic psychotic patients. Leukocytosis with or without inversion of the formula is also common because of sympathetic hyperactivation even in the absence of infection [27].

The 2017 study by Gurrera et al. [28] showed that the 2011 consensus diagnostic criteria [29] incorporated in DSM-5 were superior to those of DSM-IV [30] but required clinical validation. The DSM-IV, Nierenberg scale, and Sachdev rating scale confirmed the diagnosis of NMS in only $55 \%, 65 \%$, and $85 \%$ of our cases, respectively (Table 2). The DSM-5 consensus (Table 1) [4] does not require major and minor criteria and seems more likely to identify non-typical NMS presentations. It seemed appropriate to base the NMS diagnosis on the DSM-5 criteria in our daily practice. NMS is an exclusion diagnosis, even with well-defined diagnostic criteria [11]. Patients taking NLs may present a pathological condition unrelated to their usual drug use, and some differential diagnoses are potentially life-threatening, including meningoencephalitis, epilepsy, toxic or septic encephalopathy, malignant hyperthermia, heat stroke, serotonin syndrome, and malignant catatonia [31]. Brain scan, lumbar puncture, toxicological screening, electroencephalogram, and metabolic and infection testing may be necessary to rule out disorders that are more critical. Some differential diagnoses are less known to intensivists and require expertise and multidisciplinarity. In fact, NMS can easily be confused with other dysautonomia diseases (serotonin syndrome, malignant catatonia, or clozapine-induced hyperthermia) where rigidity, hyperpyrexia, dysautonomia, and polymedications are common. In our series, SSRIs were used in $15 \%$ of the cases at non-toxic doses in combination with NLs. Serotonin syndrome is related to selective toxicity of SSRIs but is characterized by tremor, hyper-reflexia, myoclonus, ataxia, more gastrointestinal symptoms (diarrhea, nausea, and vomiting), less rigidity, and hyperthermia [32]. Malignant catatonia is characterized by prodromal behavioral symptoms (psychosis, agitation, and catatonic excitement) and motor symptoms (dystonia, waxy flexibility, and repetitive stereotyped movements). Because catatonia symptoms are present in NMS too, distinguishing between the two is difficult [23]. Clozapine-induced hyperthermia, a known and variable side effect of clozapine, is considered a clinical presentation of NMS by some authors [33].

The severity of NMS is associated with the onset of life-threatening complications related to the physiopathology of the disease, long ICU stay, and immobility [3,31]. The Sachdev rating scale [34] allows for the diagnosis of NMS, severity assessment, and follow-up. A total score $>8$ and a score $\geq 2$ in at least three of the six categories establish the diagnosis of NMS. Other complica- 
tions not considered in the Sachdev rating scale are addressed in the organ failure scores usually used in intensive care and emergency settings, i.e., the quick-Sequential Organ Failure Assessment (SOFA) and SOFA scores. The quick-SOFA score is an easily reproducible clinical score in an emergency setting with a significant prognostic value. It includes three items: hypotension (systolic blood pressure $\leq 100 \mathrm{mmHg}$ ), high respiratory rate ( $\geq 22$ breaths/min), and altered consciousness (Glasgow coma scale score $\leq 14)$. The SOFA score is used for the severity assessment and follow-up of critically ill patients in daily practice. The Sachdev rating scale requires further clinical validation in various populations and more demanding assessment, but it could be an interesting follow-up marker in the ICU setting.

Treatment must be initiated as soon as NMS is suspected [31]. These patients require monitoring and intensive care, which cannot be provided in the psychiatric ward. Both delayed diagnostic and therapeutic management and inadequate management settings have been recognized as the prognostic factors for morbidity and mortality [3]. This observation in our practice has led to a close collaboration between the two departments of psychiatry and emergency/intensive care that involves emergency care training for psychiatrists, multidisciplinary meetings, and rigorous transfer regulation of suspected cases of NMS. Considered a prognostic factor [3], the causal psychotropic treatment was discontinued in all our patients as soon as NMS was suspected. Resuscitation measures and specific therapies are shown in Table 6 $[3,31,35,36]$. Given the rarity of NMS and the acute nature of its onset, the current recommendations are based on a low level of evidence. Randomized controlled trials are lacking, and the main treatment guidelines are based on case studies, meta-analyses, or expert opinions [36]. In addition, recommendations must be adapted to the local specificities. Dantrolene $[37,38]$ is a muscle relaxant antagonist of the ryanodine-1 receptors of striated muscles and is recommended in hypermetabolic forms of NMS (hyperthermia and rigidity). None of our patients was administered dantrolene, as it was unavailable. The central active dopaminergic agonists (bromocriptine, amantadine, or levodopa) have been reported to reduce the recovery time and mortality [38]; however, these molecules are used outside marketing authorization, and the treatment duration is not defined. ECT stimulates serotoninergic and dopaminergic neurotransmission and is currently the gold standard in cases non-responsive to specific pharmacological treatments $[11,36]$. However, the following imperatives need to be clarified: definition of pharmacological treatment failure, electrode positioning, and the intensity, frequency, and duration of sessions. Current guidelines recommend $6-10$ bilateral ECT sessions [39]. It is performed under general anesthesia, and its re- quirements include pre-anesthetic assessment, optimal anesthetic platform, vigilance, and anesthesiologist-operator communication. Some anesthetic agents should be used with caution. Sevoflurane, succinylcholine, or their combination is associated with malignant hyperthermia; hence, they should be avoided. The current availability of sugammadex makes rocuronium an interesting alternative to succinylcholine [40]. ECT is performed a tour center under sedation without intubation, but none of our patients received it as a treatment for NMS.

The reintroduction of psychotropic treatment in patients who need it but are at a risk of NMS recurrence is a real dilemma for clinicians. Recurrence risk is unpredictable, and relapse rates are highly variable [41]. When reintroduction is considered, the following recommendations $[2,42]$ are essential to minimize the recurrence risk without canceling it: interval of at least 2 weeks, complete resolution of symptoms, use of different and less powerful molecules, avoiding lithium and parenteral therapies, slower titration schemes, prevention of dehydration, and close monitoring. Long-term ECT appears to be an interesting alternative. In our study, the reintroduction of NLs was performed in six patients (30\%), using atypical molecules at low doses after a therapeutic interval of 15 days. No adverse events were reported.

Mortality rates in NMS range from 5\% to 20\%; death occurs most often in the course of multivisceral failure secondary to complications of NMS, mainly renal $[2,3,13,43]$. The main prognostic factors observed are acute renal failure, respiratory failure, sepsis, advanced age, and CPK levels [2,3,13,44]. The mortality rate in our study was $10 \%$, and the deaths were related to rhabdomyolysis and renal failure. Advanced age, renal, hepatic, and hematological failure, as well as mechanical ventilation were significantly associated with mortality in our study based on the univariate analysis. Daily assessment of the SOFA score should be performed, and treatment that is more aggressive should target elderly and frail patients, especially those with limited physiological and nephronic reserves. Most episodes usually resolve within 2 weeks, but prolonged cases with residual catatonia and motor signs have been reported [42]. Recovery was longer in one of our cases, as it was complicated by meningeal hemorrhage [45]. Risk factors for the worsening of NMS include the use of conventional antipsychotic drugs and the presence of underlying structural brain pathologies. Most patients do not develop neurological sequelae, except in cases of severe hypoxia or prolonged hyperthermia [42]. This implies the importance of temperature control and optimization of oxygenation in NMS patients. These patients are considered to have brain injury, and management of secondary cerebral systemic aggressions is necessary. Interestingly, our patients' outcomes are consistent with those reported in the litera- 
Table 6. Resuscitation measures and specific therapies recommended in neuroleptic malignant syndrome $[3,31,35,36]$

\begin{tabular}{|c|c|}
\hline Category & Resuscitation and specific therapy \\
\hline \multirow[t]{6}{*}{ Conditioning and monitoring } & - Half-seated position, head at $45^{\circ}$ \\
\hline & $\begin{array}{l}\text { - Standard monitoring: heart rate and rhythm, blood pressure, oxygen saturation, temperature, and urinary } \\
\text { output }\end{array}$ \\
\hline & - Two peripheral venous lines (18-16 gauge) and central venous lines \\
\hline & $\begin{array}{l}\text { - Biology: blood count+platelet count, liver function, renal function, hemostasis, electrolytes (kalemia, cal- } \\
\text { cemia, phosphatemia, and magnesia), glycemia, C-reactive protein, arterial blood gases, lactates, urinary } \\
\text { pH, procalcitonin }\end{array}$ \\
\hline & - Nasogastric tube in case of swallowing disorder, hypersalivation, or consciousness alteration \\
\hline & - Standard chest radiography \\
\hline \multirow[t]{8}{*}{ Fluid resuscitation and renal support } & - Crystalloids: saline 0.9\%, Ringer's lactate solution \\
\hline & -3-6 L/24 hr or more+monitoring \\
\hline & - Renal objectives: urinary output 2-3 mL/kg/hr and urinary $\mathrm{pH}>6.5$ \\
\hline & - Stop vascular filling in case of oliguria or if blood volume is optimized to avoid risk of overload \\
\hline & - Avoid nephrotoxicity \\
\hline & - Avoid colloids \\
\hline & - Bicarbonates on a case-by-case basis \\
\hline & - Dialysis \\
\hline \multirow[t]{3}{*}{ Cooling } & - Ambient temperature around $23^{\circ} \mathrm{C}$ \\
\hline & - Cooling blankets and ice blocks \\
\hline & - Chest position elevated at $45^{\circ}$ from bed level \\
\hline \multirow[t]{3}{*}{ Respiratory support } & - Oxygen therapy \\
\hline & - Respiratory kinesiotherapy: postural measures, incentive spirometry, and drainage of bronchial secretions \\
\hline & - Tracheal intubation and mechanical ventilation \\
\hline \multirow[t]{2}{*}{ Agitation control } & - Avoid restraint as much as possible \\
\hline & - Benzodiazepines (lorazepam or midazolam): 1-2 mg intravenously every 4-6 hours (maximum 8 mg/day) \\
\hline \multirow{3}{*}{$\begin{array}{l}\text { Antiarrhythmic and antihypertensive } \\
\text { treatment }\end{array}$} & - Correction of hydroelectrolytic disorders \\
\hline & - Antiarrhythmic therapies \\
\hline & - Calcium inhibitors (do not combine with dantrolene) \\
\hline \multirow{4}{*}{$\begin{array}{l}\text { Prevention of complications related to } \\
\text { intensive care unit stay }\end{array}$} & - Stress ulcer prevention \\
\hline & - Pharmacologic and/or mechanical thromboembolic prophylaxis \\
\hline & $\begin{array}{l}\text { - Prevention of decubitus complications: regular position changes, anti-bedsore mattress, motor kinesio- } \\
\text { therapy, and early mobilization }\end{array}$ \\
\hline & $\begin{array}{l}\text { - Prevention of metabolic complications: energy intake based on } 5 \% \text { glucose serum with electrolytes+nu- } \\
\text { tritional management: enteral (oral or by nasogastric tube) and/or parenteral administration }\end{array}$ \\
\hline \multirow[t]{3}{*}{ Specific therapy } & $\begin{array}{l}\text { - Bromocriptine } 2.5-5 \mathrm{mg} \text { every } 8 \text { hours (oral or nasogastric tube) or amantadine } 100 \mathrm{mg} \text { every } 8 \text { hours } \\
\text { (oral or nasogastric tube) }\end{array}$ \\
\hline & - Dantrolene $1 \mathrm{mg} / \mathrm{kg}$ every 4-6 hours intravenously for 48 hours (maximum 10 mg/kg/day) \\
\hline & - Electroconvulsive therapy as second line therapy \\
\hline
\end{tabular}

ture, and no "antidotal" therapy was given to any patient. This highlights the importance of early diagnosis and supportive treatment in resource-limited settings where specific treatments are not available. However, early initiation of specific treatment, if available, may have affected the outcome of the deceased patients. Analysis of the predictive factors of mortality was not possible given the limited number of patients in the "death" group; however, through univariate analysis, this study identified the important complications that require close monitoring. The stated conclusions need to be verified using larger samples in future multi- centric studies. Another limitation of the retrospective nature of this study is the lack of long-term follow-up of all the patients.

In conclusion, NMS is a diagnostic and therapeutic neuropsychiatric emergency. It requires clinical, multidisciplinary, and dynamic expertise to avoid overlooking atypical forms or differential diagnoses. The use of more flexible diagnostic criteria is essential to detect atypical forms, which are more frequent with second-generation antipsychotics and non-psychotropic drugs. The identification of prognostic factors specific to our context could improve the management, but this would require large national 
multicentric cohorts for research. Nevertheless, advanced age, high CPK levels, and renal failure are the potential factors to be considered in future studies, and they require the clinician's full consideration during management. Finally, this study allowed us to update and contextualize our bedside procedures (Supplementary Material 1).

\section{ARTICLE INFORMATION}

\section{Ethics statement}

This study was approved by the local Institutional Review Board of Comite d'Ethique Hospitalo-Universitaire de Fes (IRB No. $16 / 21$ ), and the need for informed consent from patients was waived.

\section{Conflict of interest}

No potential conflict of interest relevant to this article.

\section{ORCID}

Soumaya Touzani

Nawfal Houari

https://orcid.org/0000-0003-0572-334X

Abderrahim Elbouazzaoui https://orcid.org/0000-0001-9477-2922

Brahim Boukatta

https://orcid.org/0000-0001-8551-5799

Nabil Kanjaa

https://orcid.org/0000-0002-0983-4680

\section{Author contributions}

Conceptualization: ST, MH, NH. Data curation: ST, MH, YYK. Formal analysis: ST, MH. Methodology: ST, NH. Project administration: ST, NH, NK. Visualization: ST, AE, BB. Writing-original draft: ST. Writing-review \& editing: all authors.

\section{Supplementary materials}

Supplementary materials can be found via https://doi.org/10. 18700/jnc.210019.

\section{REFERENCES}

1. Caroff SN, Mann SC. Neuroleptic malignant syndrome. Med Clin North Am 1993;77:185-202.

2. Shalev A, Hermesh H, Munitz H. Mortality from neuroleptic malignant syndrome. J Clin Psychiatry 1989;50:18-25.

3. Modi S, Dharaiya D, Schultz L, Varelas P. Neuroleptic malignant syndrome: complications, outcomes, and mortality. Neurocrit Care 2016;24:97-103.

4. American Psychiatric Association. DSM-5: diagnostic and statistical manual of mental disorders. 5th ed. Arlingtone, VA: American Psychiatric Publishing; 2013.
5. Gurrera RJ, Simpson JC, Tsuang MT. Meta-analytic evidence of systematic bias in estimates of neuroleptic malignant syndrome incidence. Compr Psychiatry 2007;48:205-11.

6. Gurrera RJ. A systematic review of sex and age factors in neuroleptic malignant syndrome diagnosis frequency. Acta Psychiatr Scand 2017; 135:398-408.

7. Strugnell C, Dunstan DW, Magliano DJ, Zimmet PZ, Shaw JE, Daly RM. Influence of age and gender on fat mass, fat-free mass and skeletal muscle mass among Australian adults: the Australian diabetes, obesity and lifestyle study (AusDiab). J Nutr Health Aging 2014;18:540-6.

8. Bergen SE, O'Dushlaine CT, Lee PH, Fanous AH, Ruderfer DM, Ripke S, et al. Genetic modifiers and subtypes in schizophrenia: investigations of age at onset, severity, sex and family history. Schizophr Res 2014;154:48-53.

9. Panagiotakopoulos L, Neigh GN. Development of the HPA axis: where and when do sex differences manifest? Front Neuroendocrinol 2014;35:285-302.

10. Delay J, Pichot P, Lemperiere T, Elissalde B, Peigne F. A non-phenothiazine and non-reserpine major neuroleptic, haloperidol, in the treatment of psychoses. Ann Med Psychol (Paris) 1960;118:145-52.

11. Strawn JR, Keck PE Jr, Caroff SN. Neuroleptic malignant syndrome. Am J Psychiatry 2007; 164:870-6.

12. Mihara K, Kondo T, Suzuki A, Yasui-Furukori N, Ono S, Sano A, et al. Relationship between functional dopamine D2 and D3 receptors gene polymorphisms and neuroleptic malignant syndrome. Am J Med Genet B Neuropsychiatr Genet 2003;117: 57-60.

13. Sahin A, Cicek M, Gonenc Cekic O, Gunaydin M, Aykut DS, Tatli $\mathrm{O}$, et al. A retrospective analysis of cases with neuroleptic malignant syndrome and an evaluation of risk factors for mortality. Turk J Emerg Med 2017;17:141-5.

14. Menon V, Thamizh JS, Rajkumar RP, Selvakumar N. Neuroleptic malignant syndrome (or malignant extrapyramidal autonomic syndrome): time to revisit diagnostic criteria and terminology? Aust N Z J Psychiatry 2017;51:102.

15. Su YP, Chang CK, Hayes RD, Harrison S, Lee W, Broadbent M, et al. Retrospective chart review on exposure to psychotropic medications associated with neuroleptic malignant syndrome. Acta Psychiatr Scand 2014;130:52-60.

16. Tse L, Barr AM, Scarapicchia V, Vila-Rodriguez F. Neuroleptic malignant syndrome: a review from a clinically oriented perspective. Curr Neuropharmacol 2015;13:395-406.

17. Gurrera RJ. Sympathoadrenal hyperactivity and the etiology of neuroleptic malignant syndrome. Am J Psychiatry 1999;156: 169-80. 
18. Wagner R, Fink RH, Stephenson DG. Effects of chlorpromazine on excitation-contraction oupling events in fast-twitch skeletal muscle fibres of the rat. Br J Pharmacol 2004;141:624-33.

19. Anglin RE, Rosebush PI, Mazurek MF. Neuroleptic malignant syndrome: a neuroimmunologic hypothesis. CMAJ 2010;182: E834-8.

20. Tural U, Onder E. Clinical and pharmacologic risk factors for neuroleptic malignant syndrome and their association with death. Psychiatry Clin Neurosci 2010;64:79-87.

21. Levenson JL. Neuroleptic malignant syndrome. Am J Psychiatry 1985;142:1137-45.

22. Gupta V, Magon R, Mishra BP, Sidhu GB, Mahajan R. Risk factors in neuroleptic malignant syndrome. Indian J Psychiatry 2003;45:30-5.

23. Lang FU, Lang S, Becker T, Jäger M. Neuroleptic malignant syndrome or catatonia? Trying to solve the catatonic dilemma. Psychopharmacology (Berl) 2015;232:1-5.

24. Benazzi F. Neuroleptic malignant syndrome without rigidity. Ital J Neurol Sci 1991;12:121.

25. Özdemir İ, Kuru E, Safak Y, Tulacı RG. A neuroleptic malignant syndrome without rigidity. Psychiatry Investig 2018;15:226-9.

26. Caroff SN, Mann SC, Campbell E. Atypical antipsychotics and neuroleptic malignant syndrome. Psychiatr Ann 2000;30:31421.

27. Vörös V, Osváth P, Fekete S, Tényi T. Antipsychotics and rhabdomyolysis: differential diagnosis and clinical significance of elevated serum creatine kinase levels in psychiatric practice. Psychiatr Hung 2009;24:175-84.

28. Gurrera RJ, Mortillaro G, Velamoor V, Caroff SN. A validation study of the international consensus diagnostic criteria for neuroleptic malignant syndrome. J Clin Psychopharmacol 2017; 37:67-71.

29. Gurrera RJ, Caroff SN, Cohen A, Carroll BT, DeRoos F, Francis A, et al. An international consensus study of neuroleptic malignant syndrome diagnostic criteria using the Delphi method. J Clin Psychiatry 2011;72:1222-8.

30. American Psychiatric Association. Diagnostic and statistical manual of mental disorders. 4th ed. Washington, DC: American Psychiatry Association; 1994.

31. Oruch R, Pryme IF, Engelsen BA, Lund A. Neuroleptic malignant syndrome: an easily overlooked neurologic emergency. Neuropsychiatr Dis Treat 2017;13:161-75.

32. Tormoehlen LM, Rusyniak DE. Neuroleptic malignant syndrome and serotonin syndrome. Handb Clin Neurol 2018;157: 663-75.
33. Sachdev P, Kruk J, Kneebone M, Kissane D. Clozapine-induced neuroleptic malignant syndrome: review and report of new cases. J Clin Psychopharmacol 1995;15:365-71.

34. Sachdev PS. A rating scale for neuroleptic malignant syndrome. Psychiatry Res 2005; 135:249-56.

35. Bosch X, Poch E, Grau JM. Rhabdomyolysis and acute kidney injury. N Engl J Med 2009;361:62-72.

36. Schönfeldt-Lecuona C, Kuhlwilm L, Cronemeyer M, Neu P, Connemann BJ, Gahr M, et al. Treatment of the neuroleptic malignant syndrome in international therapy guidelines: a comparative analysis. Pharmacopsychiatry 2020;53:51-9.

37. Musselman ME, Saely S. Diagnosis and treatment of drug-induced hyperthermia. Am J Health Syst Pharm 2013;70:34-42.

38. Hirjak D, Sartorius A, Kubera KM, Wolf RC. Antipsychotic-induced motor symptoms in schizophrenic psychoses-Part 2: catatonic symptoms and neuroleptic malignant syndrome. Nervenarzt 2019;90:12-24.

39. Hasan A, Falkai P, Wobrock T, Lieberman J, Glenthoj B, Gattaz WF, et al. World Federation of Societies of Biological Psychiatry (WFSBP) guidelines for biological treatment of schizophrenia, part 2: update 2012 on the long-term treatment of schizophrenia and management of antipsychotic-induced side effects. World J Biol Psychiatry 2013;14:2-44.

40. Kadiyala PK, Kadiyala LD. Anaesthesia for electroconvulsive therapy: an overview with an update on its role in potentiating electroconvulsive therapy. Indian J Anaesth 2017;61:373-80.

41. Mahendran R, Winslow M, Lim D. Recurrent neuroleptic malignant syndrome. Aust N Z J Psychiatry 2000;34:699-700.

42. Ware MR, Feller DB, Hall KL. Neuroleptic malignant syndrome: diagnosis and management. Prim Care Companion CNS Disord 2018;20:17r02185.

43. Nakamura M, Yasunaga $H$, Miyata $H$, Shimada T, Horiguchi $H$, Matsuda S. Mortality of neuroleptic malignant syndrome induced by typical and atypical antipsychotic drugs: a propensity-matched analysis from the Japanese Diagnosis Procedure Combination database. J Clin Psychiatry 2012;73:427-30.

44. Taniguchi N, Tanii H, Nishikawa T, Miyamae Y, Shinozaki K, Inoue $\mathrm{Y}$, et al. Classification system of complications in neuroleptic malignant syndrome. Methods Find Exp Clin Pharmacol 1997;19:193-9.

45. Jouali T, Boukatta B, Bechri B, Houari N, Bouazzaoui A, Sbai H, et al. A neuroleptic malignant syndrome complicated by subarachnoid hemorrhage and revealing cerebral vasculitis. Pan Afr Med J 2014;19:247. 